\title{
A cohesive finite element formulation for modelling fracture and delamination in solids
}

\author{
S ROY CHOWDHURY and R NARASIMHAN \\ Department of Mechanical Engineering, Indian Institute of Science, Bangalore \\ 560012 , India \\ e-mail: narasi@mecheng.iisc.ernet.in
}

\begin{abstract}
In recent years, cohesive zone models have been employed to simulate fracture and delamination in solids. This paper presents in detail the formulation for incorporating cohesive zone models within the framework of a large deformation finite element procedure. A special Ritz-finite element technique is employed to control nodal instabilities that may arise when the cohesive elements experience material softening and lose their stress carrying capacity. A few simple problems are presented to validate the implementation of the cohesive element formulation and to demonstrate the robustness of the Ritz solution method. Finally, quasi-static crack growth along the interface in an adhesively bonded system is simulated employing the cohesive zone model. The crack growth resistance curves obtained from the simulations show trends similar to those observed in experimental studies.
\end{abstract}

Keywords. Cohesive zone models; fracture; delamination; finite elements.

\section{Introduction}

During the span of the last two decades, considerable research work in the field of fracture mechanics has been devoted to the analysis of stationary cracks in various structural systems. While such an analysis is useful to predict the behaviour of a system till the point of initiation of crack propagation, its applicability reduces during the propagation phase. In many cases, such as homogeneous ductile materials, layered structures, composites etc., initiation of crack growth does not indicate catastrophic failure. A stable crack extension phase, which is associated with a steady increase in the external load or crack driving force, precedes catastrophic failure in such systems. In other words, in these cases, there is reserve strength in the system even after crack initiation has occurred and it is expended during propagation. Thus, design considerations call for detailed analyses and understanding of the crack growth phase to profitably make use of this reserve strength.

Several investigators have contributed in providing an understanding of the mechanics and the practical implications of stable crack growth by using both analytical and numerical techniques. However, primarily due to the difficulty involved in the treatment of the governing equations, analytical solutions have mostly been limited to idealised cases, 
such as, elastic-perfectly plastic homogeneous materials (Chitaley \& McClintock 1971; Drugan et al 1982) or materials with linear hardening (Amazigo \& Hutchinson 1977). The early finite element studies (Rice \& Sorensen 1978; Malluck \& King 1980; Sham 1983; Narasimhan et al 1987) have extensively used the nodal release procedure to simulate quasi-static crack growth as well as dynamic crack propagation. In this method, crack extension is assumed to take place when a fracture criterion, based on a critical stress or deformation measure near the crack tip, is satisfied. In the finite element procedure, the boundary condition at the crack tip node is then replaced by the point load acting on it. This point load is subsequently reduced to zero in several increments, at the end of which a traction-free element surface emerges, and the crack advances by one element length.

Despite widespread uses, a clear defect in the nodal release technique is the lack of a material length scale. This implies that it is not possible to incorporate both the requirements of critical stress and fracture energy for simulating crack growth or interface debonding. Further, the absence of a material length scale leads to strong dependence of the finite element results on the size of the elements used near the crack tip. Consequently, in recent times, a few formulations have been suggested to overcome the above mentioned difficulties. These include a cohesive zone model proposed by Needleman (1987), a computational void-cell approach by Xia et al (1995) and a virtual internal bond model by Gao \& Klein (1998). In this paper, attention is focussed on the cohesive zone model (CZM) which was originally suggested by Needleman (1987) in order to simulate the process of inclusion debonding from a metal matrix. CZM, also called embedded process zone (EPZ) approach, is based on the cohesive zone concepts of Barenblatt (1962) and Dugdale (1960) and is a purely continuum formulation. This approach has been successfully employed in various numerical investigations including crack growth analysis in homogeneous ductile materials (Tvergaard \& Hutchinson 1992), interface debonding (Tvergaard \& Hutchinson 1993), impact damage in brittle materials (Camacho \& Ortiz 1996), analysis of sandwiched structures (Tvergaard \& Hutchinson 1994; Lin et al 1997; Kolhe et al 1999; Roychowdhury \& Narasimhan 2000b) etc.

However, the fundamental equations of this approach, the corresponding finite element formulation, and the special technique necessary to solve the resulting algebraic equations have not been reported in a systematic manner in the literature. In view of the large potential of this method in terms of range of applicability, a detailed description of these aspects of CZM is very important and constitutes the main focus of the present paper. Further, a few numerial examples are presented in order to validate the finite element formulation of CZM and to demonstrate the robustness of the special technique to solve the algebraic equations. Finally, as an example of the scope of application of CZM, a brief description of numerical simulations of stable crack growth in polymeric adhesive joints is presented.

\section{Traction-separation law}

In CZM, the potential crack propagation plane or the cleavage plane (for example, a bimaterial interface) is idealised as a cohesive zone or cohesive interface and is assumed to support a nominal traction field $\mathbf{T}$ (force/unit reference area). This traction field, in general, has components both normal and tangential to the cohesive interface. The mechanical response of the cohesive interface is described through a constitutive law (in terms of a potential function) relating the traction field $\mathbf{T}$ with a separation parameter. The constitutive equations are such that, with increasing interfacial separation, the traction across the 
interface reaches a maximum, decreases and eventually vanishes so that complete decohesion occurs. It should be emphasized here that this constitutive description is a continuum one, and thus does not represent the interaction of two individual atoms across the interface. Also, it does not account for discrete dislocation effects. Consequently, the functional dependence of the traction field on interface separation is not uniquely determined.

A number of various functions have been proposed to describe the traction-separation law for the cohesive interface. Needleman (1987) employed a polynomial function for this constitutive law to simulate inclusion debonding. Camacho \& Ortiz (1996) made use of a linear relationship with negative slope between the interface traction and separation in their simulations of impact damage in brittle solids. A trapezoidal law has been assumed in various numerical investigations (Tvergaard \& Hutchinson 1992-1994; Kolhe et al 1999; Roychowdhury \& Narasimhan 2000a). For the purpose of definiteness, attention is restricted to the trapezoidal constitutive relationship between the traction components and interface separation in this work. This law is described in the following. However, it must be emphasized that the cohesive finite element formulation to be presented in $\S 3$ is general and any traction-separation law can be employed along with it.

Let $A$ and $B$ be two initially coincident material points on the plane ahead of the crack tip (see figure 1a). Let $A^{\prime}$ and $B^{\prime}$ denote their corresponding positions in the deformed configuration, as shown in figure $1 \mathrm{~b}$. Let $u_{n}$ and $u_{t}$ be the normal and tangential components of the separation between points $A^{\prime}$ and $B^{\prime}$. A non-dimensional effective separation parameter $\lambda$ is defined as,

$$
\lambda=\left(\left(\frac{u_{n}}{\delta_{n}^{c}}\right)^{2}+\left(\frac{u_{t}}{\delta_{t}^{c}}\right)^{2}\right)^{1 / 2},
$$

where, $\delta_{n}^{c}$ and $\delta_{t}^{c}$ are critical values of $u_{n}$ and $u_{t}$ for the case of pure normal and tangential separation respectively. In the undeformed configuration $\lambda$ takes the value zero, since points $A$ and $B$ coincide with each other. As the interface separates, $\lambda$ increases in magnitude and attains a value of unity when complete separation occurs.

The dependence of the nominal traction vector across the interface on the separation parameter $\lambda$ is implicitly represented through a potential $\phi\left(u_{n}, u_{t}\right)$ which is defined by:

$$
\phi\left(u_{n}, u_{t}\right)=\delta_{n}^{c} \int_{0}^{\lambda} \sigma(\lambda) \mathrm{d} \lambda,
$$

such that the work of separation per unit undeformed area of the interface $\Gamma_{\circ}$ is given by the value of $\phi$ for $\lambda=1$. The function $\sigma(\lambda)$ in (2) is prescribed a priori. The specific variation of $\sigma(\lambda)$ assumed in this work is shown in figure 1c. The physical significance of the function $\sigma(\lambda)$ can be appreciated by considering the special case of pure normal separation $\left(u_{t}=0\right)$. For this case, the nominal traction component normal to the interface $T_{n}=\sigma(\lambda)$, where $\lambda=u_{n} / \delta_{n}^{c}$. Thus, it can be seen from figure 1c, that in this case, the normal traction increases monotonically as $\lambda$ increases from 0 to $\lambda_{1}$ and attains a maximum value of $\hat{\sigma}$. It then remains constant over the range $\lambda_{1}$ to $\lambda_{2}$, and finally drops to zero as $\lambda \rightarrow 1$. Thus, $\hat{\sigma}$ represents the strength of the interface under pure normal separation.

In a general case involving both normal and tangential separation, the respective components of the nominal traction vector across the interface are given by:

$$
\bar{T}_{n}=\frac{\partial \phi}{\partial u_{n}}=\frac{\sigma(\lambda)}{\lambda} \frac{u_{n}}{\delta_{n}^{c}}
$$


(a)

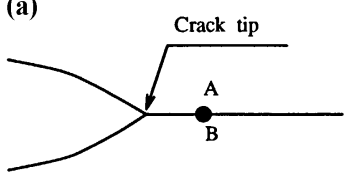

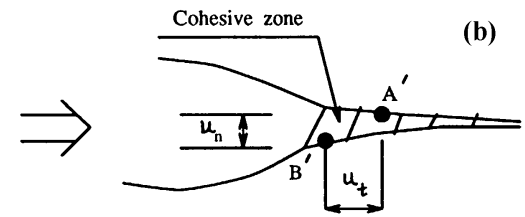

(c)

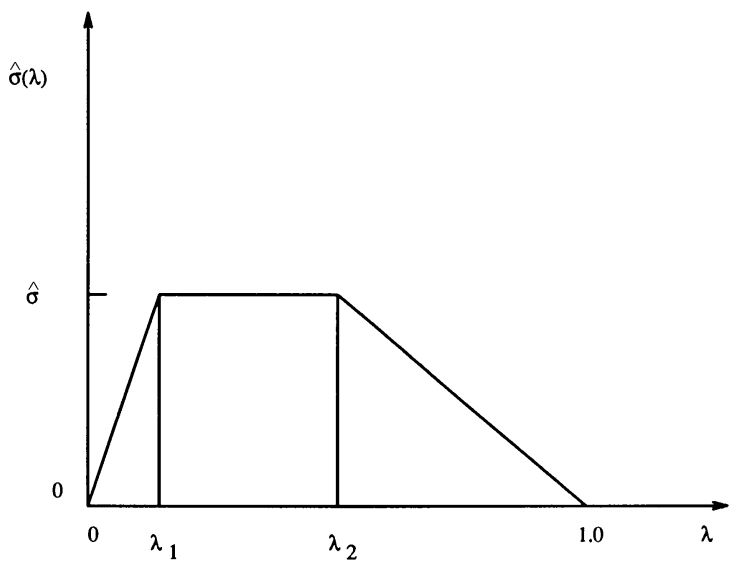

Figure 1. (a) Undeformed, (b) deformed configuration of the region near a crack tip or debond zone, and (c) The prescribed traction-separation law for the cohesive interface.

and

$$
\bar{T}_{t}=\frac{\partial \phi}{\partial u_{t}}=\frac{\sigma(\lambda)}{\lambda} \frac{u_{t}}{\delta_{t}^{c}} \frac{\delta_{n}^{c}}{\delta_{t}^{c}}
$$

In the special case of a pure tangential separation $\left(u_{n}=0\right)$, the tangential traction is given by $\bar{T}_{t}=\left(\delta_{n}^{c} / \delta_{t}^{c}\right) \sigma(\lambda)$ where $\lambda=u_{t} / \delta_{t}^{c}$. Thus, the strength of the interface under pure shear separation is $\left(\delta_{n}^{c} / \delta_{t}^{c}\right) \hat{\sigma}$. It should be noted here that the use of the potential ensures that the work of separation per unit undeformed area is $\Gamma_{\circ}$ irrespective of the combination of normal and tangential displacements across the cohesive zone. Further, it must be emphasized that the definition of the potential itself via (2) to (4) is possible only if nominal traction and normal and tangential separation components with respect to the undeformed configuration are employed (see figure 1b). The work of separation per unit undeformed area of the interface is given by the area under the traction versus separation curve shown in figure $1 \mathrm{c}$. Thus,

$$
\Gamma_{\circ}=\frac{1}{2} \hat{\sigma} \delta_{n}^{c}\left[1-\lambda_{1}+\lambda_{2}\right] .
$$

The parameters governing the separation law are therefore the work of separation $\Gamma_{0}$, the peak stress quantity $\hat{\sigma}$ and the ratio of critical displacements $\delta_{n}^{c} / \delta_{t}^{c}$. These may be specified based on atomistic models of separation (see, for example, Tadmor et al 1996) or on a phenomenological basis depending on whether the separation process is governed by a ductile void coalescence or a brittle cleavage mechanism (Tvergaard \& Hutchinson 1994; Kolhe et al 1999). Further, since the separation process is governed by both energy and stress criteria, a natural length scale appears in the model. The significance of this characteristic length parameter is further discussed in $\S 6$. The factors $\lambda_{1}$ and $\lambda_{2}$ govern the shape of the separation function. The studies conducted by Tvergaard \& 
Hutchinson (1994) suggest that the details of the shape of the separation law are relatively unimportant. The choice of different parameters in the traction-separation law is discussed in $\S \S 5$ and 6 .

\section{Finite element formulation for CZM}

In order to account for the material and geometric nonlinearity that arise in mechanics problems of finite deformation, a Lagrangian formulation is employed and the finite element equations are derived from the incremental form of the principle of virtual work (see, for example, Hibbitt et al 1970, McMeeking \& Rice 1975). However, it is necessary to modify this principle in order to represent the mechanical response of the cohesive interface. In this work, the cohesive interface is also discretized along with the remaining part of the continuum and an element formulation is derived from the modified virtual work principle.

A body that is in static equilibrium at a time $t$ during the deformation history is considered. The body is assumed to occupy a configuration $V_{t}$ in the current state and a configuration $V_{o}$ in the reference state (see figure $2 \mathrm{a}$ ). The position vector of a material particle is denoted as $X_{i}$ and $x_{i}$ in the reference and current configurations respectively, with respect to a fixed Cartesian frame with base vectors $\mathbf{e}_{1}, \mathbf{e}_{2}$ and $\mathbf{e}_{3}$. The broken lines in figure 2 a represent an interface. The tangent vector $\mathbf{t}^{o}$ is oriented such that material of part \# 1 is on the left side, as the interface is traversed along the direction of $\mathbf{t}^{o}$, and of part \# 2 is on the right side. Thus the unit normal to the interface $\mathbf{n}^{o}$ points into part $\# 1$ and away from part \# 2. Let $A$ and $B$ be two material points which in the undeformed configuration touch each other and are on either side of the interface (i.e., $A$ is in part \# 1 and $B$ is in part \# 2). Their position vectors in $V_{o}$ are denoted as $\mathbf{X}^{A}=\mathbf{X}^{B}$. After deformation, their position vectors are $\mathbf{x}^{A}$ and $\mathbf{x}^{B}$, respectively (see figure $2 \mathrm{a}$ ).

The displacement vectors can be written as

$$
\begin{aligned}
& \mathbf{u}^{A}=\mathbf{x}^{A}-\mathbf{X}^{A}, \\
& \mathbf{u}^{B}=\mathbf{x}^{B}-\mathbf{X}^{B} .
\end{aligned}
$$

The relative displacement vector of points $A$ and $B$ is given by,

$$
\Delta \mathbf{u}^{A B}=\mathbf{u}^{A}-\mathbf{u}^{B}=\mathbf{x}^{A}-\mathbf{x}^{B},
$$

since $\mathbf{X}^{A}=\mathbf{X}^{B}$. The tangential and normal components of $\Delta \mathbf{u}^{A B}$ with respect to $\mathbf{t}^{o}$ and $\mathbf{n}^{o}$ are

$$
\begin{aligned}
u_{t} & =\Delta \mathbf{u}^{A B} \cdot \mathbf{t}^{o}, \\
u_{n} & =\Delta \mathbf{u}^{A B} \cdot \mathbf{n}^{o} .
\end{aligned}
$$

Here, $u_{t}$ and $u_{n}$ are respectively the tangential and normal separation of point $A$ with respect to point $B$, where the tangent and normal are referred to the undeformed configuration $V_{o}$ (see figure $2 \mathrm{a}$ ). For consistency, the nominal traction vector $\overline{\mathbf{T}}$ across the interface is also based on the undeformed configuration (see also Needleman 1987). This interface traction vector represents the force per unit undeformed area which part \# 1 exerts on part \# 2. The tangential and normal components of the interface traction vector are given by,

$$
\begin{aligned}
\bar{T}_{t} & =\overline{\mathbf{T}} \cdot \mathbf{t}^{o}, \\
\bar{T}_{n} & =\overline{\mathbf{T}} \cdot \mathbf{n}^{o} .
\end{aligned}
$$




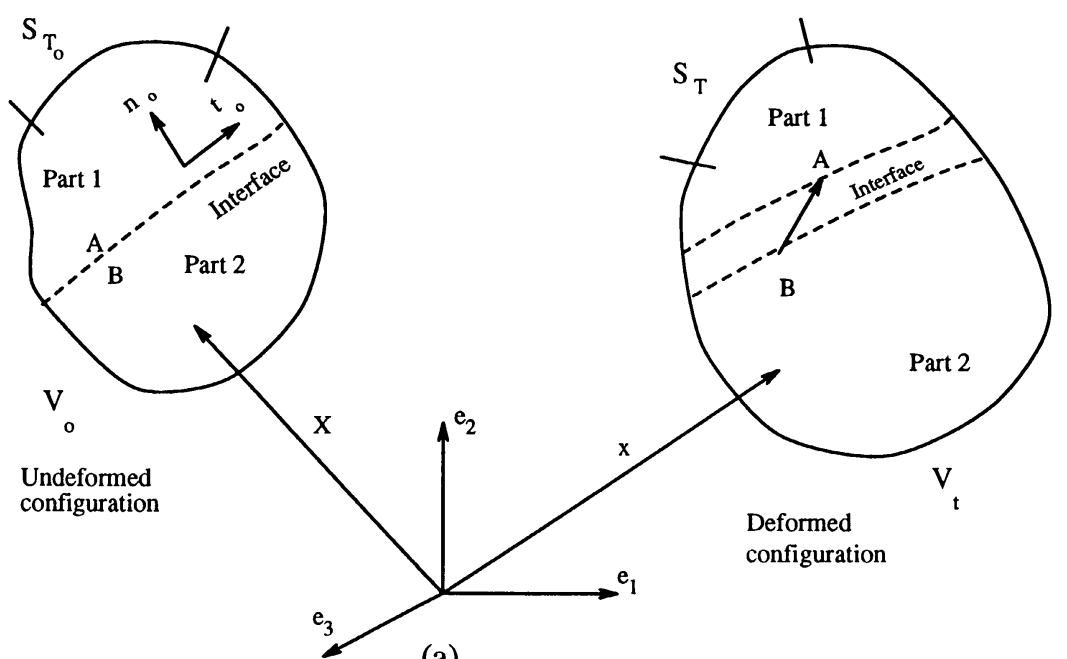

(a)

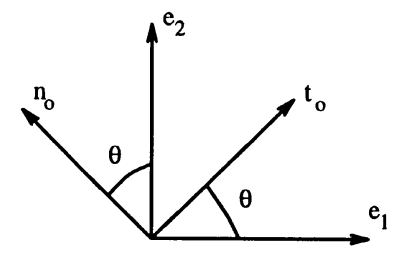

(b)

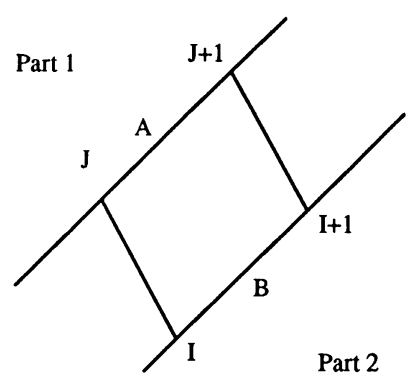

(c)

Figure 2. (a) Undeformed and deformed configuration of a continuum with a cohesive interface. (b) Transformation of axes. (c) A typical cohesive element.

The traction components $\bar{T}_{t}$ and $\bar{T}_{n}$ are considered to be functions of $u_{t}$ and $u_{n}$ (see (3) and (4)), such that,

$$
\begin{aligned}
& \bar{T}_{t}=f_{1}\left(u_{t}, u_{n}\right), \\
& \bar{T}_{n}=f_{2}\left(u_{t}, u_{n}\right) .
\end{aligned}
$$

In the present work, a Lagrangian finite element procedure is employed. In this formu clation, the reference configuration is taken to coincide with the undeformed configuration. For this choice of reference configuration, the relationship between Kirchhoff stress $\tau_{i j}$ and Cauchy stress $\sigma_{i j}$, and their Jaumann rates $^{1}$ are given by,

$$
\tau_{i j}=J \sigma_{i j}
$$

and

$$
\tau_{i j}^{*}=J\left(\sigma_{i j}^{*}+\sigma_{i j} D_{k k}\right),
$$

\footnotetext{
${ }^{1}$ Note that the Jaumann rate of $\tau_{i j}$, defined by $\tau_{i j}^{*}=\dot{\tau}_{i j}+\tau_{i k} W_{k j}-W_{i k} \tau_{k j}$ where $W_{i j}$ is the spin rate (skew symmetric part of spatial gradient of velocity), is an objective rate (see Malvern 1969).
} 
where $J=\operatorname{det}\left(\nabla_{\mathbf{X}} \mathbf{x}\right)$ is the Jacobian of the deformation. Further, $D_{i j}=\left(\partial v_{i} / \partial x_{j}+\right.$ $\left.\partial v_{j} / \partial x_{i}\right) / 2$, denotes the rate of deformation tensor. On considering the presence of the interface, the incremental form of the virtual work rate principle is given as (see Needleman 1987):

$$
\begin{aligned}
& \Delta t \int_{V_{o}}\left[\tau_{i j}^{*}-\left(\tau_{i k} D_{k j}+D_{i k} \tau_{k j}\right)\right] \delta D_{i j} \mathrm{~d} V_{o}+\Delta t \int_{V_{o}} \tau_{i j} v_{k, j} \delta v_{k, i} \mathrm{~d} V_{o} \\
& +\left\{\Delta t \int_{S_{o}^{\text {int }}}\left[\left(\dot{\bar{T}}_{n} \delta \dot{u}_{n}+\dot{\bar{T}}_{t} \delta \dot{u}_{t}\right] \mathrm{d} S_{o}^{i n t}\right\}=\Delta t \int_{V_{o}} \dot{\bar{b}}_{j} \delta v_{j} \mathrm{~d} V_{o}+\Delta t \int_{S_{T}^{o}} \dot{\bar{T}}_{j} \delta v_{j} \mathrm{~d} S_{o}\right. \\
& +\left[\int_{V_{o}} \bar{b}_{j} \delta v_{j} \mathrm{~d} V_{o}+\int_{S_{T}^{o}} \bar{T}_{j} \delta v_{j} \mathrm{~d} S_{o}-\int_{V_{o}} \tau_{i j} \delta D_{i j} \mathrm{~d} V_{o}-\int_{S_{o}^{i n t}}\left(\bar{T}_{n} \delta \dot{u}_{n}+\bar{T}_{t} \delta \dot{u}_{t}\right) \mathrm{d} S_{o}^{i n t}\right] .
\end{aligned}
$$

Here, all integrations are performed over the volume $V_{o}$ and the boundary segment $S_{T}^{o}$ in the undeformed configuration. In particular, the interface terms are integrated over interface segment $S_{o}^{\text {int }}$ in the undeformed configuration. Further, $v_{k, i}=\partial v_{k} / \partial x_{i}$ is the spatial gradient of particle velocity vector and $\dot{\bar{b}}_{j}$ and $\dot{\bar{T}}_{j}$ represent nominal body force and surface traction rates.

The term within square brackets on the right hand side of (13) is an equilibrium correction term that vanishes when the known state at time $t$ is an exact equilibrium state. The virtual work rate term due to interface traction is included on the left hand side of (13) because $\bar{T}_{t}$ and $\bar{T}_{n}$ are traction components acting on part \#2, whereas $u_{t}$ and $u_{n}$ are tangential and normal displacements of point $A$ relative to point $B$ (see figure 2a). It should be noted that in deriving (13) the virtual work principle is applied strictly in a rate sense and, thus, the time step size $\Delta t$ in this equation should be considered very small.

In this work, rate-independent elastic-plastic constitutive equations which satisfy material objectivity are considered and are assumed to have the following structure:

$$
\sigma_{i j}^{*}=L_{i j k l} D_{k l},
$$

where, $L_{i j k l}$ is the elastic-plastic constitutive tensor. In view of (12) relating the Jaumann rate of Kirchhoff and Cauchy stress tensors, (14) can be written as:

$$
\tau_{i j}^{*}=M_{i j k l} D_{k l},
$$

where, $M_{i j k l}$ is given by:

$$
M_{i j k l}=J\left(L_{i j k l}+\sigma_{i j} \delta_{k l}\right),
$$

and $\delta_{i j}$ represents the Kronecker delta. On substituting from (15) into the variational principle equation (13), and following standard procedures, the finite element equation corresponding to (13) can be obtained as,

$$
\Delta t[K]\{\dot{u}\}=\Delta t\left\{\dot{F}_{b}\right\}+\Delta t\left\{\dot{F}_{t}\right\}+\left[\left\{F_{b}\right\}+\left\{F_{t}\right\}-\{P\}-\left\{P^{i n t}\right\}\right] .
$$

Here, $[K]$ is the stiffness matrix and $\left\{\dot{F}_{b}\right\}$ and $\left\{\dot{F}_{t}\right\}$ are force rate vectors due to body force and surface traction respectively. The expressions for the element stiffness matrix (without contribution from the interface terms) and element force rate vectors are given by McMeeking \& Rice (1975). The term in square brackets on the right hand side of (17) is the equilibrium correction term (or out-of-balance force term). The element version of the 
internal force vector $\{P\}$ which occurs on the right hand side of (17) is given by

$$
\{P\}^{e}=\int_{V_{o}^{e}}[B]^{T}\{\tau\} \mathrm{d} V_{o}^{e},
$$

where [B] is the usual strain-displacement matrix involving spatial gradients of shape functions (see McMeeking \& Rice 1975). In the following, the contributions to the element stiffness matrix $\left[K^{i n t}\right]^{e}$ and element internal force vector $\left\{P^{i n t}\right\}^{e}$ from the interface are derived. If an element has no node on the interface, it does not contribute to these terms.

On differentiating (10), the nominal traction rates can be obtained as,

$$
\left\{\begin{array}{c}
\dot{\bar{T}}_{t} \\
\dot{\bar{T}}_{n}
\end{array}\right\}=\left[\begin{array}{ll}
\partial f_{1} / \partial u_{t} & \partial f_{1} / \partial u_{n} \\
\partial f_{2} / \partial u_{t} & \partial f_{2} / \partial u_{n}
\end{array}\right]\left\{\begin{array}{l}
\dot{u}_{t} \\
\dot{u}_{n}
\end{array}\right\}=[S]\left\{\begin{array}{c}
\dot{u}_{t} \\
\dot{u}_{n}
\end{array}\right\} .
$$

The last term on the left hand side of (13), which is the interface term, can be written in matrix notation as

$$
\int_{S_{o}^{\text {int }}}\left[\dot{\bar{T}}_{t} \delta \dot{u}_{t}+\dot{\bar{T}}_{n} \delta \dot{u}_{n}\right] \mathrm{d} S_{o}^{i n t}=\int_{S_{o}^{i n t}}\left\langle\delta \dot{u}_{t} \delta \dot{u}_{n}\right\rangle\left\{\begin{array}{c}
\dot{\bar{T}}_{t} \\
\dot{\bar{T}}_{n}
\end{array}\right\} \mathrm{d} S_{o}^{i n t} .
$$

On substituting (19) into (20) one obtains,

$$
\int_{S_{o}^{\text {int }}}\left[\dot{\bar{T}}_{t} \delta \dot{u}_{t}+\dot{\bar{T}}_{n} \delta \dot{u}_{n}\right] \mathrm{d} S_{o}^{\text {int }}=\int_{S_{o}^{\text {int }}}\left\langle\delta \dot{u}_{t} \delta \dot{u}_{n}\right\rangle[S]\left\{\begin{array}{l}
\dot{u}_{t} \\
\dot{u}_{n}
\end{array}\right\} \mathrm{d} S_{o}^{i n t} .
$$

If the tangent vector along the interface $\mathbf{t}_{o}$ makes an angle $\theta$ with the base vector $\mathbf{e}_{\mathbf{1}}$ (as shown in figure $2 b$ ), the tangential and normal components of the relative displacement rate can be written as:

$$
\left\{\begin{array}{l}
\dot{u}_{t} \\
\dot{u}_{n}
\end{array}\right\}=\left[\begin{array}{cc}
\cos \theta & \sin \theta \\
-\sin \theta & \cos \theta
\end{array}\right]\left\{\begin{array}{c}
\Delta \dot{u}_{1}^{A B} \\
\Delta \dot{u}_{2}^{A B}
\end{array}\right\}=[Q]\left\{\begin{array}{c}
\Delta \dot{u}_{1}^{A B} \\
\Delta \dot{u}_{2}^{A B}
\end{array}\right\} .
$$

Here, the Cartesian components of the relative displacement rate are defined by,

$$
\begin{aligned}
& \Delta \dot{u}_{1}^{A B}=\dot{u}_{1}^{A}-\dot{u}_{1}^{B}, \\
& \Delta \dot{u}_{2}^{A B}=\dot{u}_{2}^{A}-\dot{u}_{2}^{B} .
\end{aligned}
$$

In matrix form (23) can be written as,

$$
\left\{\begin{array}{c}
\Delta \dot{u}_{1}^{A B} \\
\Delta \dot{u}_{2}^{A B}
\end{array}\right\}=\left[\begin{array}{cccc}
1 & 0 & -1 & 0 \\
0 & 1 & 0 & -1
\end{array}\right]\left\{\begin{array}{c}
\dot{u}_{1}^{A} \\
\dot{u}_{2}^{A} \\
\dot{u}_{1}^{B} \\
\dot{u}_{2}^{B}
\end{array}\right\}=[A]\left\{\begin{array}{c}
\dot{u}_{1}^{A} \\
\dot{u}_{2}^{A} \\
\dot{u}_{1}^{B} \\
\dot{u}_{2}^{B}
\end{array}\right\} .
$$

On substituting (24) and (22) into (21) one obtains,

$$
\begin{aligned}
\int_{S_{o}^{i n t}} & {\left[\dot{\bar{T}}_{t} \delta \dot{u}_{t}+\dot{\bar{T}}_{n} \delta \dot{u}_{n}\right] \mathrm{d} S_{o}^{i n t} } \\
= & \int_{S_{o}^{\text {int }}}\left\langle\delta \dot{u}_{1}^{A} \delta \dot{u}_{2}^{A} \delta \dot{u}_{1}^{B} \delta \dot{u}_{2}^{B}\right\rangle[A]^{T}[Q]^{T}[S][Q][A]\left\{\begin{array}{c}
\dot{u}_{1}^{A} \\
\dot{u}_{2}^{A} \\
\dot{u}_{1}^{B} \\
\dot{u}_{2}^{B}
\end{array}\right\} \mathrm{d} S_{o}^{i n t} .
\end{aligned}
$$


In a similar way, it can be shown that the last term on the right hand side of (13) is given by,

$$
\begin{gathered}
\int_{S_{o}^{\text {int }}}\left[\bar{T}_{t} \delta \dot{u}_{t}+\bar{T}_{n} \delta \dot{u}_{n}\right] \mathrm{d} S_{o}^{i n t}=\int_{S_{o}^{\text {int }}}\left\langle\delta \dot{u}_{t} \delta \dot{u}_{n}\right\rangle\left\{\begin{array}{c}
\bar{T}_{t} \\
\bar{T}_{n}
\end{array}\right\} \mathrm{d} S_{o}^{i n t} \\
=\int_{S_{o}^{\text {int }}}\left\langle\delta \dot{u}_{1}^{A} \delta \dot{u}_{2}^{A} \delta \dot{u}_{1}^{B} \delta \dot{u}_{2}^{B}\right\rangle[A]^{T}[Q]^{T}[Q]\left\{\begin{array}{c}
\bar{T}_{1} \\
\bar{T}_{2}
\end{array}\right\} \mathrm{d} S_{o}^{i n t} .
\end{gathered}
$$

On considering a typical cohesive element as shown in figure 2c,

$$
\left\{\begin{array}{c}
\dot{u}_{1}^{A} \\
\dot{u}_{2}^{A} \\
\dot{u}_{1}^{B} \\
\dot{u}_{2}^{B}
\end{array}\right\}=\left[\begin{array}{cccccccc}
N_{J} & 0 & N_{J+1} & 0 & 0 & 0 & 0 & 0 \\
0 & N_{J} & 0 & N_{J+1} & 0 & 0 & 0 & 0 \\
0 & 0 & 0 & 0 & N_{I} & 0 & N_{I+1} & 0 \\
0 & 0 & 0 & 0 & 0 & N_{I} & 0 & N_{I+1}
\end{array}\right]\left\{\begin{array}{c}
\dot{u}_{1, J} \\
\dot{u}_{2, J} \\
\dot{u}_{1, J+1} \\
\dot{u}_{2, J+1} \\
\dot{u}_{1, I} \\
\dot{u}_{2, I} \\
\dot{u}_{1, I+1} \\
\dot{u}_{2, I+1}
\end{array}\right\},
$$

where $N_{J}=N_{I}$ and $N_{J+1}=N_{I+1}$ represent the interpolation functions defined on the undeformed configuration. On employing the above expression in (25), the stiffness matrix for the cohesive element can be obtained as,

$$
\left[K^{\text {int }}\right]^{e}=\int_{S_{o}^{\text {int }}}[N]^{T}[A]^{T}[Q]^{T}[S][Q][A][N] \mathrm{d} S_{o}^{\text {int }},
$$

where $[N]$ is the matrix of shape functions which is given on the right hand side of (27). Similarly, the internal force vector due to cohesive element traction is derived from (26) and noting that $[Q]^{T}[Q]=[I]$ as,

$$
\left\{P^{\text {int }}\right\}^{e}=\int_{S_{o}^{\text {int }}}[N]^{T}[A]^{T}\left\{\begin{array}{l}
\bar{T}_{1} \\
\bar{T}_{2}
\end{array}\right\} \mathrm{d} S_{o}^{\text {int }} .
$$

It should be noted that since the above integrands are evaluated based on undeformed configuration, only the matrix $[S]$ in (28) and $\bar{T}_{1}, \bar{T}_{2}$ in (29) change with loading. Equations (28) and (29) form the basis for the finite element formulation of the cohesive element and have been conveniently implemented as an element subroutine in the general purpose package FEAP (Taylor 1979).

\section{Ritz-finite element solution procedure}

It should be recalled from $\S 2$ that the mechanical response of the cohesive interface shows softening behaviour prior to complete separation (see figure 1c). This causes a deterioration of local stiffness in the corresponding cohesive elements and may lead to decrease in the external (or boundary) load and displacement. This phenomenon may be illustrated through some simple systems involving linear and nonlinear springs. To this end, a linear and a nonlinear spring having load versus displacement characteristics, as shown in figures $3 \mathrm{a}$ 


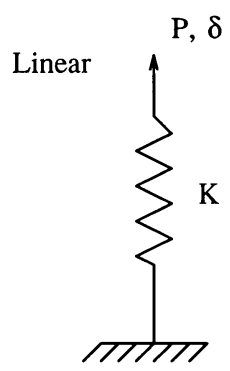

$\mathrm{P}, \delta$

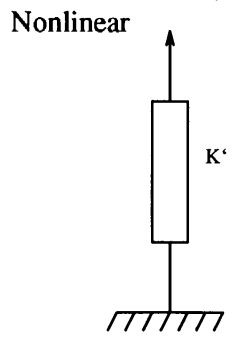

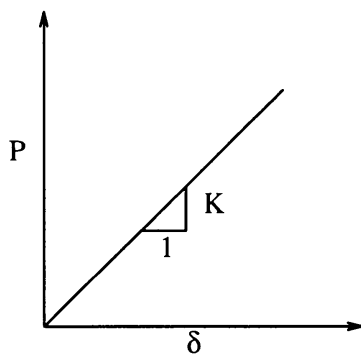

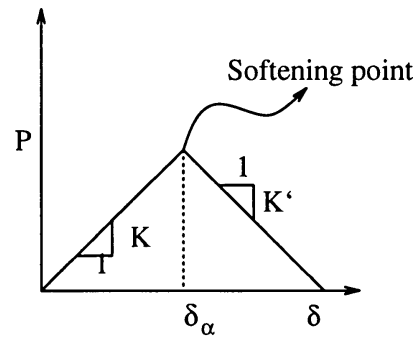

Figure 3. The load-displacement characteristics for a linear and nonlinear spring considered in the example problems.

and $\mathrm{b}$ respectively, are considered. As can be seen from figure $3 \mathrm{~b}$, the nonlinear spring has a softening branch with slope $K^{\prime}$ for displacement $\delta>\delta_{a}$. This typifies the behaviour of a cohesive element when it loses its stress carrying capacity.

The load-displacement characteristic of a system, in which the above two springs are connected in parallel, is shown in figure 4. Here, the dashed line pertains to a case with $\left|K^{\prime}\right|<K$, while the fully solid line corresponds to $\left|K^{\prime}\right|>K$. It can be observed from the figure that, if $\left|K^{\prime}\right|<K$, there is no softening in the overall load versus displacement behaviour. On the other hand, when $\left|K^{\prime}\right|>K$, there is softening in a certain part of the load versus displacement curve.

As a second example, a linear spring with stiffness $K$ which is coupled with the parallel spring system of figure 4 , as shown in figure $5 \mathrm{a}$, is considered. The load-displacement
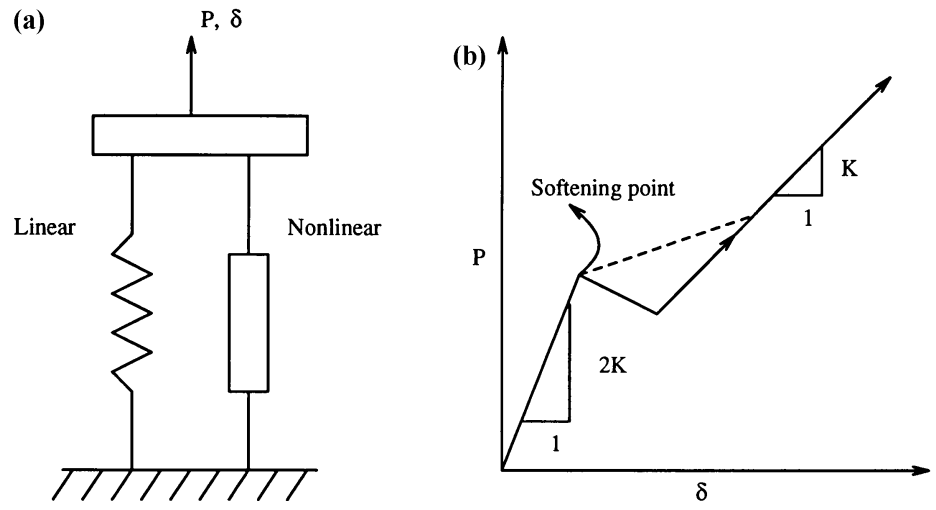

Figure 4. (a) A parallel system comprising the linear and nonlinear springs shown in figure 3, and (b) its load-displacement response. 

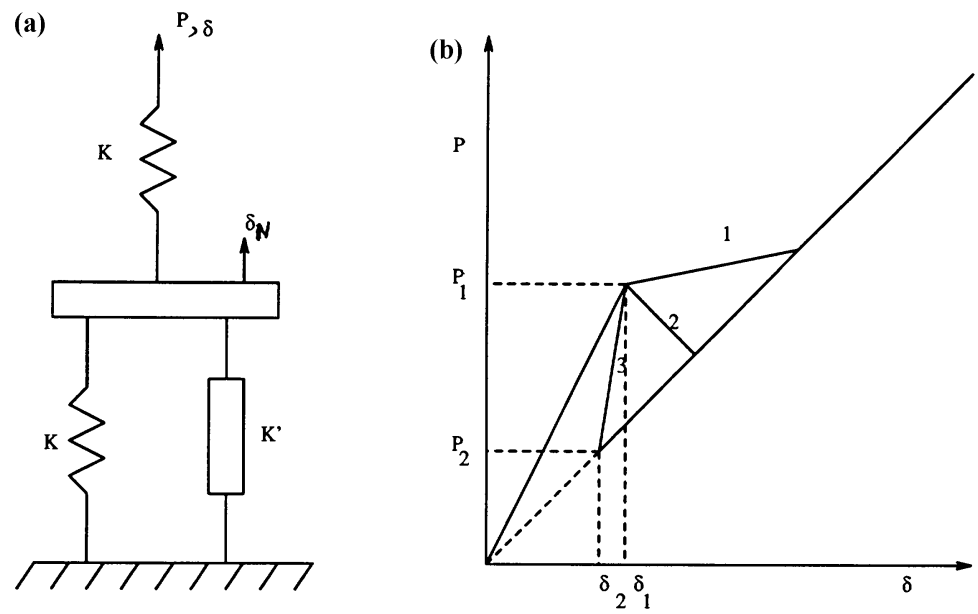

Figure 5. (a) The parallel spring system of figure 4 placed in series with the linear spring shown in figure 3. (b) The load-displacement response of the system of figure 5a.

curves corresponding to three cases are shown in figure $5 \mathrm{~b}$. The curve marked 1 pertains to $\left|K^{\prime}\right|<K$. In this case, both the load and the load point displacement increase monotonically for the entire range of the solution. The curve marked 2 in figure $5 \mathrm{~b}$ corresponds to $\left|K^{\prime}\right|$ marginally larger than $K$. As can be seen from this figure, softening behaviour is observed in the load versus displacement curve for this case. Finally, curve 3 in figure $5 \mathrm{~b}$ pertains to $\left|K^{\prime}\right| \gg K$. In this case, the load-displacement curve shows both softening and unstable behaviour. This corresponds to the branch where both $P$ and $\delta$ decrease. Hence, the load-displacement curve for this case cannot be obtained by prescribing either $P$ or $\delta$ in the usual finite element procedure. It should be noted that this situation may not be attained in any actual physical system. Instead, failure of the nonlinear spring (or the cohesive element that it represents) is expected to take place as a dynamic event. The unstable behaviour of the system is only a mathematical artifact. However, in order to solve the problem as a succession of static equilibrium states, it is essential to capture the unstable branch of the load-displacement curve.

It is possible to meet the above requirement by specifying the elongation $\delta_{N}$ of the nonlinear spring (see figure 5a) instead of the overall load $P$ or displacement $\delta$. This is because $\delta_{N}$ increases monotonically for the complete range of solutions as shown in figure 6. The overall load $P$ and $\delta$ are treated as unknowns to be solved from the equations of static equilibrium. The conventional finite element solution procedure is inadequate in this regard. An elegant way to achieve the above objective is to employ a combined Ritzfinite element method (Needleman 1982). This method is described below in detail.

The finite element equation for rate equilibrium, (17), can be written in the form,

$$
\underbrace{[K]}_{n \times n} \underbrace{\{\dot{u}\}}_{n \times 1}=\dot{\rho} \underbrace{\left\{F^{o}\right\}}_{n \times 1}+\underbrace{\{R\}}_{n \times 1},
$$

where, $\dot{\rho}\left\{F^{o}\right\}=\left\{\dot{F}^{e x t}\right\}$ is the external nodal force rate applied during the time step from $t \rightarrow t+\Delta t$, and $\{R\}=\left\{F^{e x t}\right\}-\{P\}-\left\{P^{\text {int }}\right\}$ represents the out-of-balance force at time $t$. It is important to note that in (30), the external force rate is expressed in terms of a single 

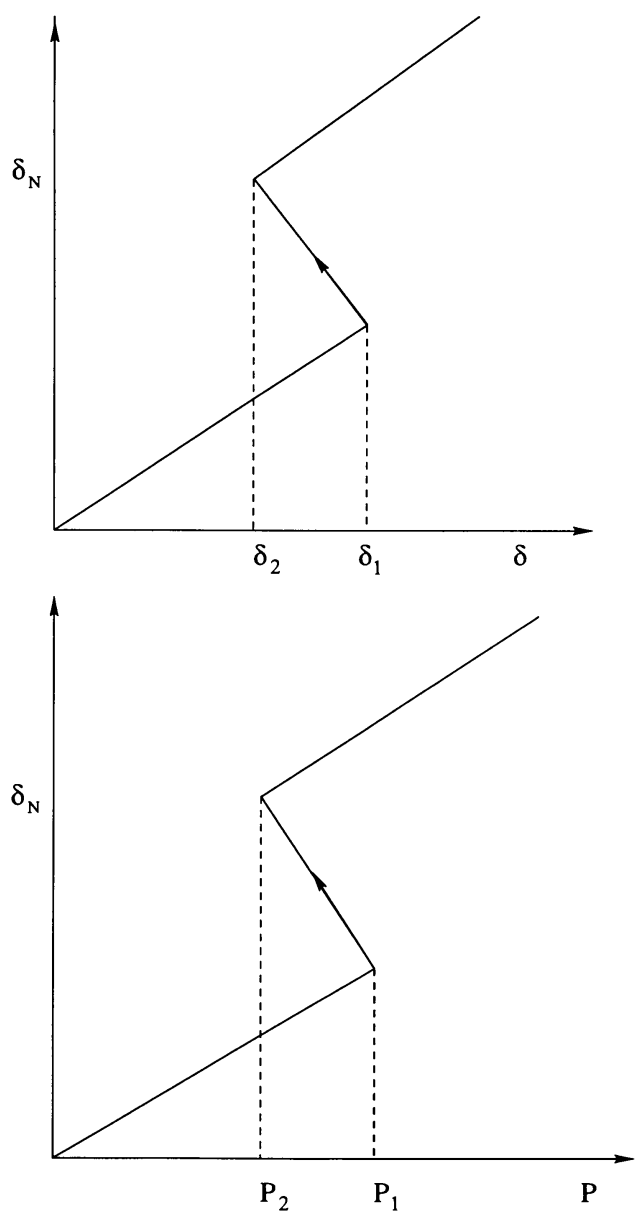

Figure 6. Displacement of the nonlinear spring $\delta_{N}$ versus overall displacement $\delta$ and load $P$ for the system shown in figure $5 \mathrm{a}$.

load rate parameter $\dot{\rho}$. In the context of interface crack growth under small-scale yielding conditions, which is considered in $\S 6$, the increment in the remote elastic stress intensity factor represents this parameter $\dot{\rho}$.

Let ' $m$ ' displacement degrees of freedom, which pertain to the nodes in the immediate vicinity of the debonded zone on the cohesive interface, be chosen to be controlled. These ' $m$ ' variables to be prescribed are placed at the bottom of the $\{\dot{u}\}$ array, so that (30) can be written in partitioned form as:

$$
\left[\begin{array}{cc}
{\left[K_{a a}\right]} & {\left[K_{a b}\right]} \\
{\left[K_{b a}\right]} & {\left[K_{b b}\right]}
\end{array}\right]\left\{\begin{array}{c}
\dot{u}_{1} \\
\dot{u}_{2} \\
\vdots \\
\frac{\dot{u}_{n-m}}{\dot{u}_{n-m+1}} \\
\vdots \\
\dot{u}_{n}
\end{array}\right\}=\dot{\rho}\left\{F^{o}\right\}+\{R\} .
$$


In the following, the steps that are followed in Ritz method are described.

- Step 1: Reordering $[K]$ matrix

Let $\dot{u}_{n-m+1}, \dot{u}_{n-m+2}, \ldots, \dot{u}_{n}$ be considered as prescribed variables. Then, (31) is modified as follows,

$$
\left[\begin{array}{c|ccc}
{\left[K_{a a}\right]} & {[0]} \\
\hline & 1 & \cdots & 0 \\
{[0]} & \vdots & \ddots & \vdots \\
& 0 & \cdots & 1
\end{array}\right]\left\{\begin{array}{c}
\dot{u}_{1} \\
\dot{u}_{2} \\
\vdots \\
\frac{\dot{u}_{n-m}}{\dot{u}_{n-m+1}} \\
\vdots \\
\dot{u}_{n}
\end{array}\right\}=\dot{\rho}\left\{\begin{array}{c}
F_{1}^{o} \\
F_{2}^{o} \\
\vdots \\
\frac{F_{n-m}^{o}}{0} \\
\vdots \\
0
\end{array}\right\}+\left\{\begin{array}{c}
R_{1} \\
R_{2} \\
\vdots \\
\frac{R_{n-m}}{0} \\
\vdots \\
0
\end{array}\right\}
$$

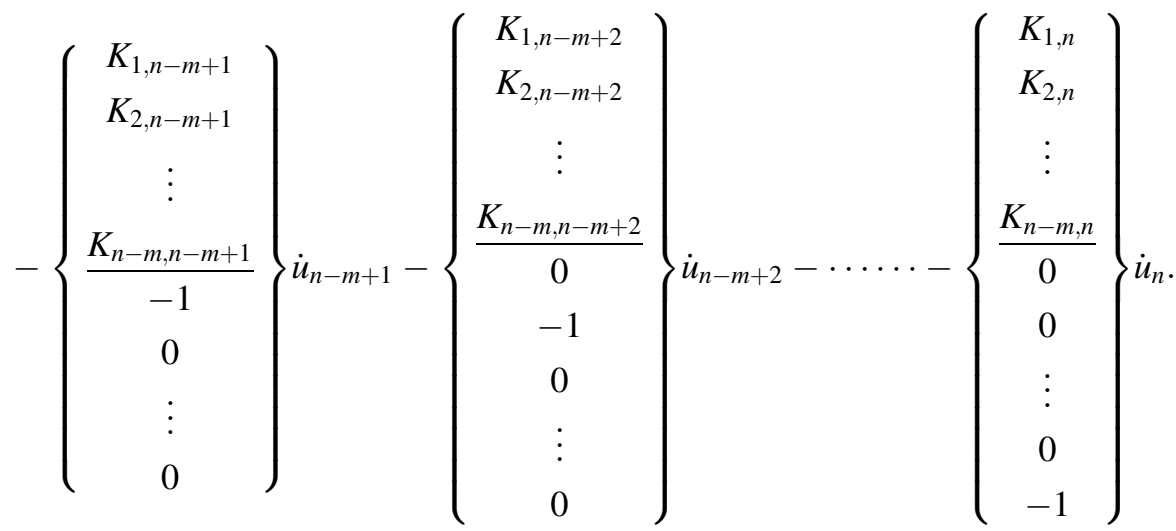

It must be noted that the last ' $m$ ' equations in (32) are identically satisfied. The above equation can be written as:

$$
\underbrace{\left[K^{\prime}\right]}_{n \times n} \underbrace{\{\dot{u}\}}_{n \times 1}=\dot{\rho} \underbrace{\{r\}^{(m+1)}}_{n \times 1}+\underbrace{\{r\}^{(m+2)}}_{n \times 1}+\dot{u}_{n-m+1} \underbrace{\{r\}^{(1)}}_{n \times 1} \cdots+\dot{u}_{n} \underbrace{\{r\}^{(m)}}_{n \times 1},
$$

where,

$$
\left[K^{\prime}\right]=\left[\begin{array}{c|ccc}
{\left[K_{a a}\right]} & & {[0]} & \\
\hline & 1 & \cdots & 0 \\
{[0]} & \vdots & \ddots & \vdots \\
& 0 & \cdots & 1
\end{array}\right],
$$


and,

$$
\begin{aligned}
\{r\}^{(m+1)} & =\left\{\begin{array}{c}
F_{1}^{o} \\
F_{2}^{o} \\
\vdots \\
F_{n-m}^{o} \\
0 \\
\vdots \\
0
\end{array}\right\} ;\{r\}^{(m+2)}=\left\{\begin{array}{c}
R_{1} \\
R_{2} \\
\vdots \\
\frac{R_{n-m}}{0} \\
\vdots \\
0
\end{array}\right\} ; \\
\{r\}^{(1)} & =-\left\{\begin{array}{c}
K_{1, n-m+1} \\
K_{2, n-m+1} \\
\vdots \\
\frac{K_{n-m, n-m+1}}{-1} \\
0 \\
\vdots \\
0
\end{array}\right\} \text { etc. }
\end{aligned}
$$

- Step 2: Computing Ritz vectors

The Ritz vectors $\{v\}^{(1)} \cdots\{v\}^{(m+2)}$ are obtained by solving the following equations:

$$
\underbrace{\left[K^{\prime}\right]}_{n \times n} \underbrace{\{v\}^{(i)}}_{n \times 1}=\underbrace{\{r\}^{(i)}}_{n \times 1} \text { for } i \in[1, m+2] .
$$

- Step 3: Formation of reduced stiffness matrix and right hand side vectors

Since (32) is a system of linear algebraic equations, the solution vector for nodal point velocities $\{\dot{u}\}$ can be written as,

$$
\underbrace{\{\dot{u}\}}_{n \times 1}=\sum_{j=1}^{(m+2)} \dot{\delta}_{j} \underbrace{\{v\}^{(j)}}_{n \times 1},
$$

where, $\dot{\delta}_{1}=\dot{u}_{n-m+1}, \dot{\delta}_{2}=\dot{u}_{n-m+2}, \ldots, \dot{\delta}_{m}=\dot{u}_{n}$. The quantities $\dot{\delta}_{m+1}$ and $\dot{\delta}_{m+2}$ can be considered as generalized displacement rates arising because of the terms $\dot{\rho}\left\{F^{o}\right\}$ and $\{R\}$ in (30).

On substituting (35) in (30) one obtains,

$$
\sum_{j=1}^{(m+2)}[K]\{v\}^{(j)} \dot{\delta}_{j}=\dot{\rho}\left\{F^{o}\right\}+\{R\} .
$$


Pre-multiplying by $\{v\}^{(i)^{T}}$ (for $i \in[1, m+2]$ ),

$$
\sum_{j=1}^{(m+2)}\{v\}^{(i)^{T}}[K]\{v\}^{(j)} \dot{\delta}_{j}=\dot{\rho}\{v\}^{(i)^{T}}\left\{F^{o}\right\}+\{v\}^{(i)^{T}}\{R\} .
$$

Let,

$$
\left.\begin{array}{l}
a_{i j}=\{v\}^{(i)^{T}}[K]\{v\}^{(j)} \\
b_{i}=\{v\}^{(i)^{T}}\left\{F^{o}\right\} \\
d_{i}=\{v\}^{(i)^{T}}\{R\}
\end{array}\right\} \quad i, j \in[1, m+2] .
$$

Then, (37) can be written in the form,

$$
\sum_{j=1}^{m+2} a_{i j} \dot{\delta}_{j}=\dot{\rho} b_{i}+d_{i}, \quad i \in[1, m+2] .
$$

\section{- Step 4: Solving reduced equations}

At this point, it is required to select the variable that is to be controlled. It should be noted here that the load rate parameter $\dot{\rho}$ is also treated as an unknown in the Ritz formulation. Among the ' $m+1$ ' unknowns $\dot{\delta}_{1}, \dot{\delta}_{2} \cdots \dot{\delta}_{m}, \dot{\rho}$, the variable which had the largest relative growth in the previous time step $(t-\Delta t) \rightarrow t$ can be prescribed during the time step $t \rightarrow(t+\Delta t)$.

Assuming that during the time step $t \rightarrow(t+\Delta t), \dot{\delta}_{k}(1 \leq k \leq m)$ is prescribed, (39) is modified as

$$
\sum_{j=1}^{k-1} a_{i j} \dot{\delta}_{j}+\left(-b_{i}\right) \dot{\rho}+\sum_{j=k+1}^{m+2} a_{i j} \dot{\delta}_{j}=-a_{i k} \dot{\delta}_{k}+d_{i}, \quad i \in[1, m+2] .
$$

The above step is equivalent to replacing the $k$ th column of matrix $[a]$ by $-\{b\}$ and the vector $\{b\}$ by the negative of the $k$ th column of matrix $[a]$. Further, $\dot{\delta}_{k}$ and $\dot{\rho}$ are interchanged. Let $[a]^{\prime}$ be the modified coefficient matrix and $\left\{\dot{\delta}^{\prime}\right\}$ be the modified unknown vector. Then, the reduced equations are:

$$
\underbrace{[a]^{\prime}}_{(m+2) \times(m+2)}\{\dot{\delta}\}^{\prime}=-\dot{\delta}_{k} \underbrace{\left\{a_{i k}\right\}}_{(m+2) \times 1}+\underbrace{\{d\}}_{(m+2) \times 1},
$$

where $\left\{a_{i k}\right\}$ is the $k$ th column of $[a]$. The value of $\dot{\delta}_{k}$ can be taken to be the same as that computed in the previous time step. It must be noted that the matrix $[a]^{\prime}$ is not symmetric. However, this does not affect the overall efficiency of the algorithm since the number of equations $(m+2)$ in (41) is usually small. The value for $\left\{\dot{\delta}^{\prime}\right\}$ is calculated by solving the system of equations given by (41) and then $\dot{\delta}_{k}$ and $\dot{\rho}$ are interchanged to obtain $\{\dot{\delta}\}$.

\section{- Step 5: Obtaining global displacement array}

Using $\{\dot{\delta}\}$ the global nodal point velocities $\{\dot{u}\}$ are obtained from (35). It must be noted that the above algorithm enables determination of the load increment $\dot{\rho}$ that is required to maintain static equilibrium, while debonding occurs along the cohesive interface. 


\section{Numerical examples}

The numerical implementation of the cohesive element model is validated by conducting single cohesive element tests in uniaxial tension and simple shear. Further, in order to illustrate the robustness of Ritz-finite element method some problems involving linear and nonlinear springs, which were described in $\S 4$, are analysed.

\subsection{Uniaxial tension test}

The mesh and boundary conditions used for uniaxial tension test of the cohesive element are shown in figure 7a. The objective of this test is to reproduce the prescribed traction versus separation law discussed in $\S 2$ under uniaxial tension. For element 1, Young's modulus $E$ and Poisson's ratio $\nu$ are taken as $10^{6}$ and 0.0 , respectively. The corresponding values for element 2 are chosen as $10^{2}$ and 0.0 . For the cohesive element, the peak stress $\hat{\sigma}$ is taken as 1.0 , and the shape parameters $\lambda_{1}$ and $\lambda_{2}$ as 0.15 and 0.5 , respectively. The critical normal displacement $\delta_{n}^{c}=0.01$ and critical tangential displacement $\delta_{t}^{c}=\delta_{n}^{c}$. The analysis is carried out by specifying the vertical displacements $\delta_{n}$ at the upper nodes of the cohesive element, until debonding occurs (i.e., till $\lambda$ attains a value of unity). It should be emphasized that the Ritz-finite element solution procedure renders such a prescription possible.

(a)

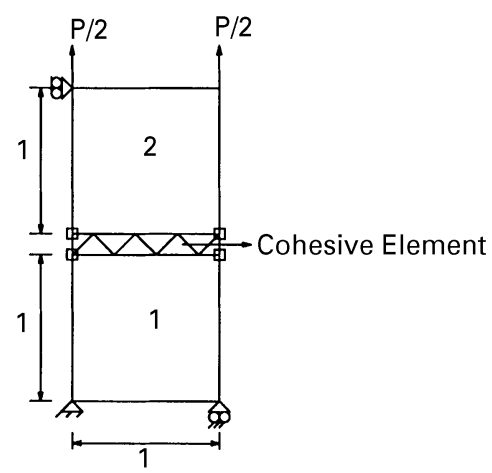

(b)

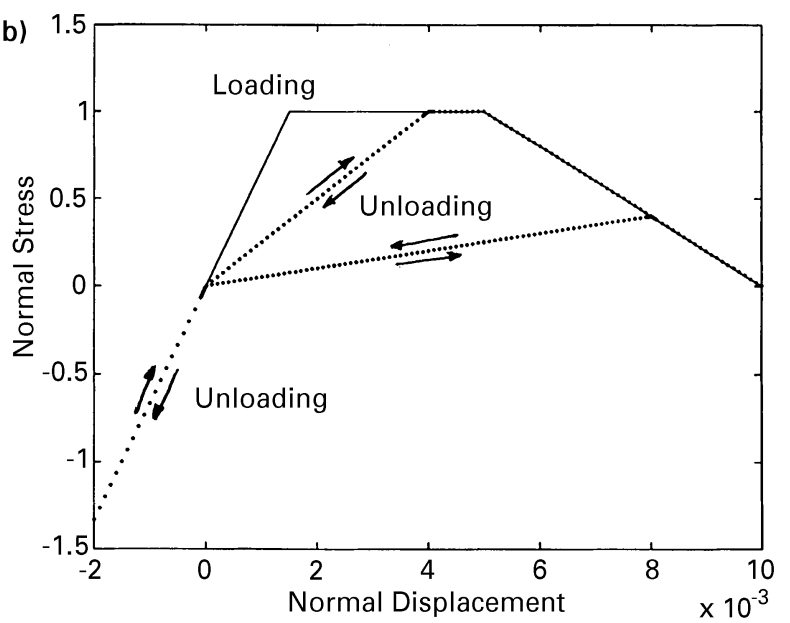

Figure 7. (a) Finite element mesh and boundary conditions used for the single cohesive element tension test. (b) Normal stress versus displacement across the interface for the tension test. 
The variation of normal stress with normal displacement, obtained from the analysis, is shown in figure $7 \mathrm{~b}$. It can be observed from this figure that the above variation follows the traction-separation law discussed in $\S 2$. The traction becomes zero when $\delta_{n}$ (normal displacement) becomes equal to the pre-set critical value $\delta_{n}^{c}$. In figure $7 \mathrm{~b}$, dotted curves pertaining to unloading and reloading at different stages are also displayed. The unloading curve traces a linear path. The reloading curve coincides with the unloading curve until $\lambda$ attains the value at the start of unloading, and thereafter follows the traction-separation curve. Under uniaxial compression, the cohesive zone behaves as a stiff spring.

\subsection{Simple shear test}

The cohesive element is also tested for debonding by specifying relative tangential displacements. The mesh and boundary conditions for the simple shear test are shown in figure 8a. The material properties chosen for elements 1 and 2 are same as in the previous example. For the cohesive element, $\hat{\sigma}, \lambda_{1}$ and $\lambda_{2}$ are taken as $1.0,0.15$ and 0.5 , respectively. The critical normal and tangential displacements are chosen as $\delta_{n}^{c}=0.001$ and $\delta_{t}^{c}=0.1$. The analysis is carried out by specifying tangential displacement on the upper surface of the cohesive element until debonding occurs. The shear stress versus tangential displacement

(a)

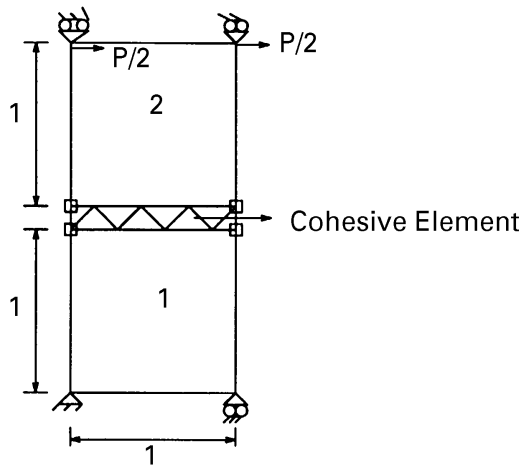

(b)

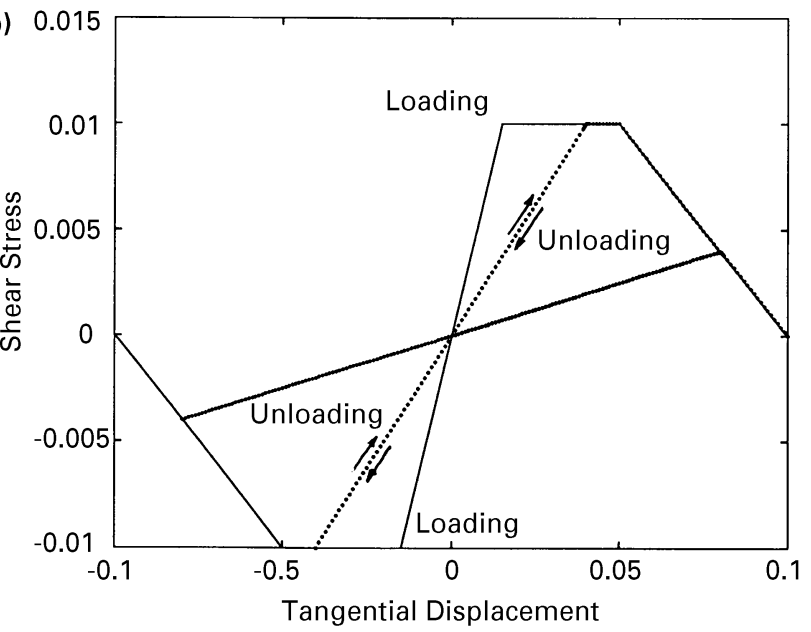

Figure 8. (a) Finite element mesh and boundary conditions used for the single cohesive element shear test. (b) Shear stress versus tangential displacement across the interface for the shear test. 
curve obtained from the analysis is shown in figure $8 \mathrm{~b}$, which follows the tractionseparation law as prescribed. The traction becomes zero when tangential displacement equals the critical value $\delta_{t}^{c}$. On reversing the direction of the tangential displacement, the cohesive traction changes sign. The unloading and reloading paths are traced in a similar way as in the uniaxial tension test (see dotted lines in figure 8b).

\subsection{Validation problems involving nonlinear springs}

In order to illustrate the robustness of the Ritz-finite element method in solving problems with softening or unstable behaviour, the examples involving linear and nonlinear springs, presented in $\S 4$, are considered.

5.3a Springs in parallel: This example pertains to figure 4, where the linear and nonlinear springs are placed in parallel. In order to model the above problem with finite elements, it should first be noted that a cohesive element obeying the traction-separation law shown in figure 1c behaves like the nonlinear spring of figure $3 \mathrm{~b}$, if $\lambda_{1}$ and $\lambda_{2}$ are taken to be equal. Figure 9 a shows the mesh used for analysing the problem with normal load $P$ applied on the top surface. Here, element 4 is a linear elastic element that simulates the

(a)

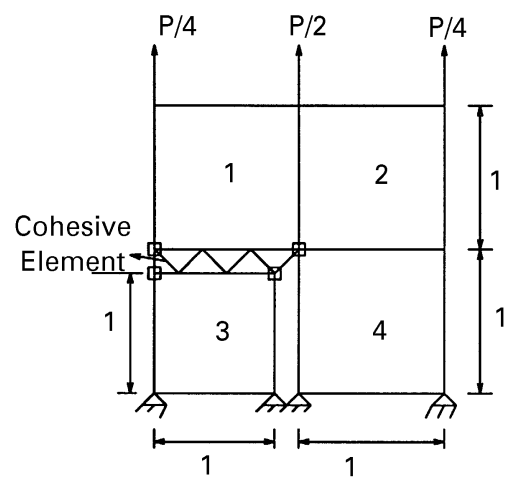

(b)

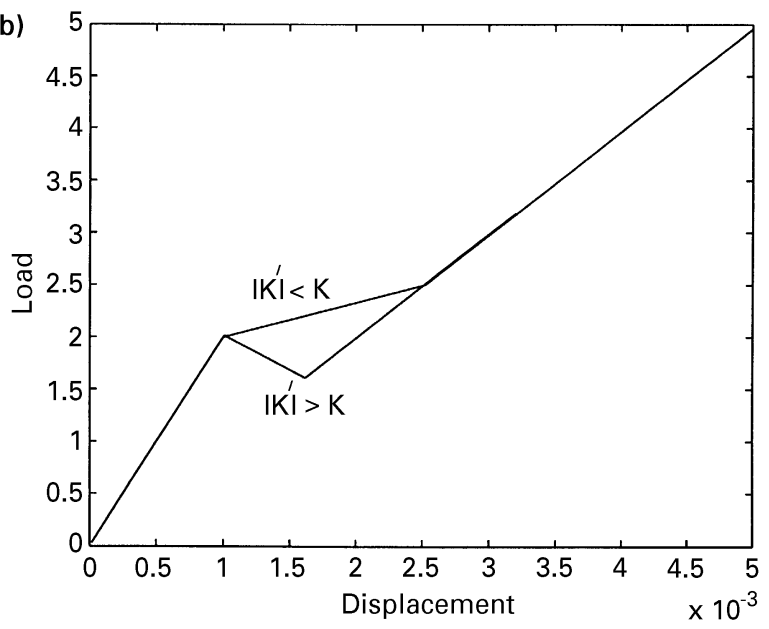

Figure 9. (a) Finite element model of the parallel spring system depicted in figure 4. (b) Load-displacement response obtained from the finite element solution. 
linear spring in figure 4 a with stiffness $K=1000$, while the nonlinear spring is represented by the cohesive element. Elements 1,2 and 3 are taken to be rigid with fictitiously high Young's modulus.

In order to simulate the case $\left|K^{\prime}\right|<K$, the cohesive zone parameters are taken as $\hat{\sigma}=1.0, \delta_{n}^{c}=\delta_{t}^{c}=0.0025, \lambda_{1}=\lambda_{2}=0.4$. For this choice of parameters, the stiffness of the nonlinear spring (cohesive element) is $K=1000$ when $\lambda$ is less than $\lambda_{1}$ and is $K^{\prime}=-666.7$, when $\lambda$ is greater than $\lambda_{1}$. For modelling the case $\left|K^{\prime}\right|>K$, the cohesive element properties are taken as, $\hat{\sigma}=1.0, \delta_{n}^{c}=\delta_{t}^{c}=0.0016, \lambda_{1}=\lambda_{2}=0.625$. Thus, the stiffness of the nonlinear spring is $K=1000$ when $\lambda$ is less than $\lambda_{1}$ and is $K^{\prime}=-1666.7$ when $\lambda$ exceeds $\lambda_{1}$. In both cases, normal displacements on the upper edge of the cohesive element are prescribed and the Ritz method is employed to obtain the solution. The load versus displacement curves for the two cases are shown in figure 9b. These curves match well with the expected analytical solutions which are displayed qualitatively in figure $4 \mathrm{~b}$.

5.3c Combination of linear and nonlinear springs: Figure 10a shows the finite element model for the spring system shown in figure 5a. The elements 1,2 and 3 are taken as rigid with a fictitiously high Young's modulus, while for elements 4 and $5, E=10^{3}$ and 500, respectively, and $\nu=0$. As in the previous example, element 4 and the cohesive element

(a)

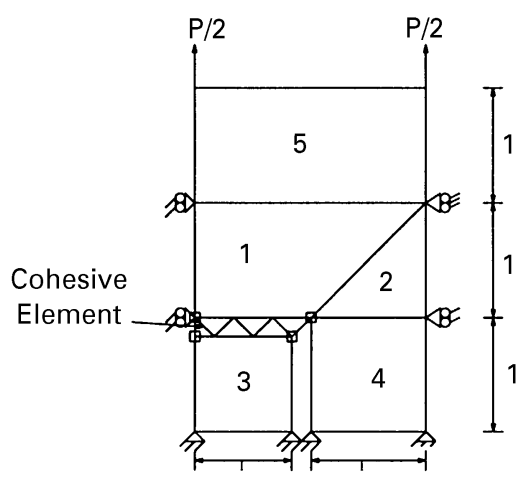

(b)

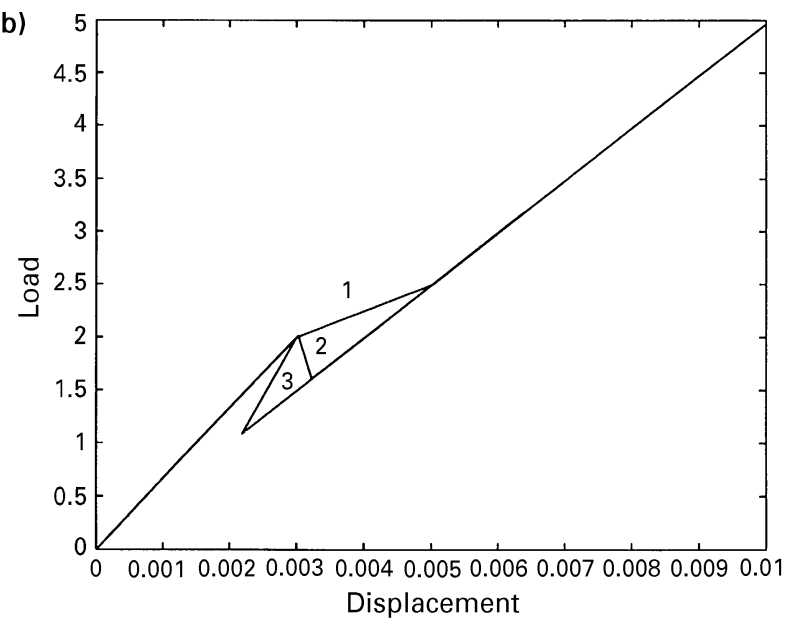

Figure 10. (a) Finite element model of the system involving linear and nonlinear springs depicted in figure 5a. (b) Load-displacement response obtained from the finite element solution. 
represent respectively, the linear and nonlinear springs in parallel (see figure 5a). Element 5 is another linear spring in series with the above parallel system as in figure 5a. The problem is solved by specifying the normal displacements on the upper edge of the cohesive element and using the Ritz method. The load versus displacement curves obtained from the analysis are shown in figure $10 \mathrm{~b}$. The following three cases are considered.

In the first case, which corresponds to $\left|K^{\prime}\right|<K$, the cohesive element properties are taken as $\hat{\sigma}=1, \delta_{n}^{c}=\delta_{t}^{c}=0.0025$, and $\lambda_{1}=\lambda_{2}=0.4$. For this choice of material properties, the stiffness of the nonlinear spring is $K=1000$ for $\lambda<\lambda_{1}$, and is $K^{\prime}=-666.7$ for $\lambda>\lambda_{1}$. In the second case, $K^{\prime}$ is marginally greater than $K$. The cohesive element properties for this case are $\hat{\sigma}=1.0, \delta_{n}^{c}=\delta_{t}^{c}=0.0016$ and $\lambda_{1}=\lambda_{2}=0.625$. Thus, the stiffness of the nonlinear spring is $K=1000$ for $\lambda \leq \lambda_{1}$, and is $K^{\prime}=-1666.7$ for $\lambda>\lambda_{1}$. In order to model the case $\left|K^{\prime}\right| \gg K$, the cohesive element properties are taken as, $\hat{\sigma}=1$, $\delta_{n}^{c}=\delta_{t}^{c}=0.0011$, and $\lambda_{1}=\lambda_{2}=0.9091$. For this choice, the stiffness of the nonlinear spring is $K=1000$ for $\lambda<\lambda_{1}$ and is $K^{\prime}=-10000.0$, for $\lambda>\lambda_{1}$. The load-displacement curves corresponding to the three cases are marked as 1, 2 and 3 in figure 10b. These curves match well with the expected analytical solutions which are shown qualitatively in figure $5 \mathrm{~b}$. In particular, it must be noted that the Ritz method reproduces the softening and unstable branch of the solution in case 3 , wherein both the overall load $P$ and displacement $\delta$ decrease. This would be impossible to achieve in a direct finite element solution procedure.

\section{Simulation of stable crack growth in adhesive joints}

A thin ductile polymeric adhesive layer is often employed for bonding two components in applications such as space technology, microelectronic packaging, aerospace and automobile industry. Experimental evidence has shown that interfacial fracture is a very common failure mode in such sandwiched layer geometry (see, for example, Chai 1988, 1992, Chai \& Chiang 1996, Swadener \& Liechti 1998). In their experiments conducted on ductile polymeric joints, Chai \& Chiang (1996) observed that the fracture of adhesive bonds is characterized by stable crack propagation followed by catastrophic growth. Further, the fracture energy increases considerably with increasing layer thickness, particularly for mode II loading (Chai 1988). This enhancement is attributed to plastic deformation in the adhesive layer. In order to understand the above phenomenon, Roy Chowdhury \& Narasimhan (2000a) carried out numerical simulations of stable crack growth in adhesively bonded joints using the cohesive element formulation discussed in $\S 3$. A brief presentation of these simulation details and salient results is made below.

\subsection{Modelling aspects}

A system comprising two identical semi-circular elastic adherends, joined by a thin polymeric adhesive layer of thickness $h$ is considered (see figure 11). A semi-infinite crack lies at the interface between the adhesive layer and the top adherend. Plane strain, smallscale yielding conditions are assumed to prevail. The ratio of the Young's modulus of the elastic adherends to that of the adhesive layer, $E_{s} / E$, is assumed to be 20 , which is representative of a joint system comprising the aluminium adherends and a typical epoxy based adhesive. The Poisson's ratio of the adherends, $\nu_{s}$, is taken as 0.3 .

A circular domain with radius $R \approx 15000 h$ is modelled with four-noded isoparametric quadrilateral finite elements. An overall view of a typical finite element discretization is 


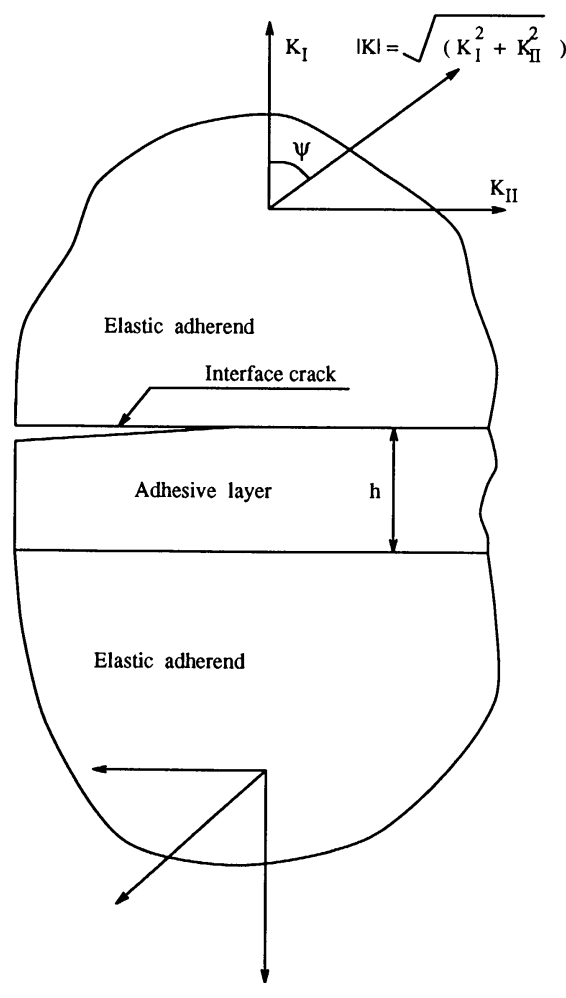

Figure 11. Schematic of the small scale yielding boundary value problem for simulating interface crack growth in adhesively bonded joints.

shown in figure 12a. An enlarged view of the region near the interface between the adhesive layer and the elastic adherends is displayed in figure $12 \mathrm{~b}$. Figure $12 \mathrm{c}$ shows the details of the highly refined mesh in the region close to the crack tip. The uniform mesh region ahead of the tip shown in this figure is used to model crack growth. The dimension $l$ of the smallest elements in figure $12 \mathrm{c}$ is $0.1 R_{o}$, where $R_{o}$ is an estimate of the plastic zone size at the initiation of crack propagation (see $\S 6.2$, (46) for definition). A set of Cartesian axes $X_{1}, X_{2}$, established with origin $O$ at the crack tip in the undeformed configuration, is shown in figure $12 \mathrm{c}$.

The displacement field corresponding to the dominant term of the mixed mode solution for a cracked linear elastic solid, involving the mode I and mode II stress intensity factors $K_{I}$ and $K_{I I}$ (see Kanninen \& Popelar 1985), is specified as the boundary condition on the outermost boundary $S$. The simulations are carried out for different combinations of normalised layer thickness $h / R_{o}$, and the mode mixity parameter $\psi=\tan ^{-1}\left(K_{I I} / K_{I}\right)$. All plastic deformation in the adhesive layer is confined within the active region shown in figure $12 \mathrm{a}$, which has a radius of $R / 20$, so that small-scale yielding conditions are preserved. The constant stiffness of the elastic region surrounding the active mesh is statically condensed using a ring-by-ring static condensation method.

The crack propagation process is simulated by using the cohesive zone model discussed in $\S 2$ to represent the interface ahead of the crack tip. The finite element formulation for the cohesive zone presented in $\S 3$ is employed. The cohesive elements lose their stress carrying capacity when the non-dimensional effective separation parameter $\lambda$ (see (1) for definition) reaches a unit value. This gives rise to a pair of new traction-free surfaces and the crack propagates by the length $l$ of the element. 
(a)

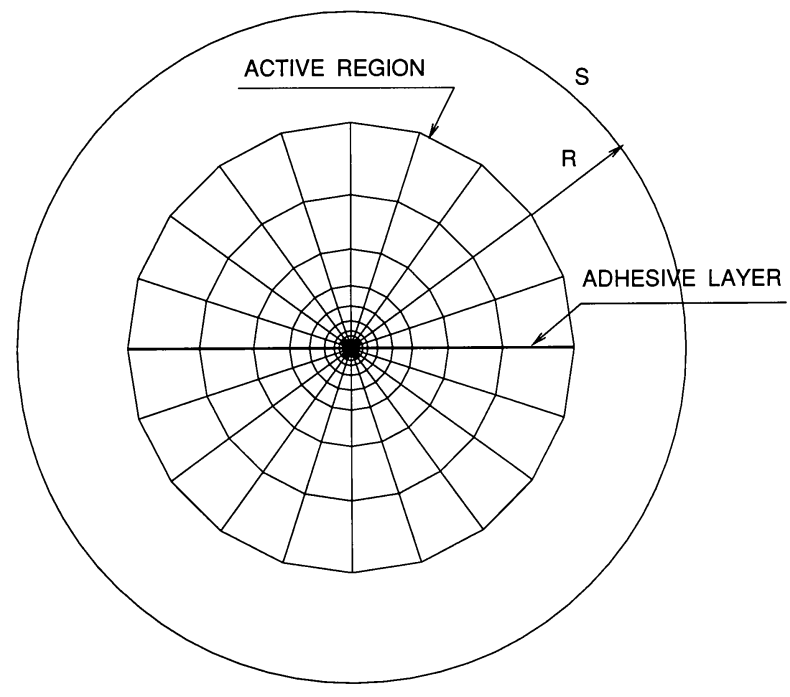

(b)
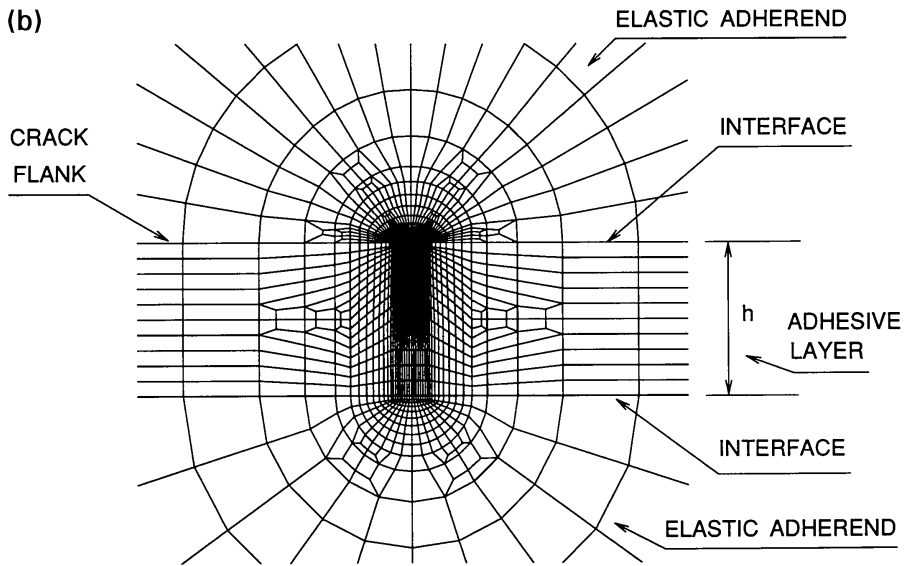

(c) $\quad \mathrm{X}_{2}$

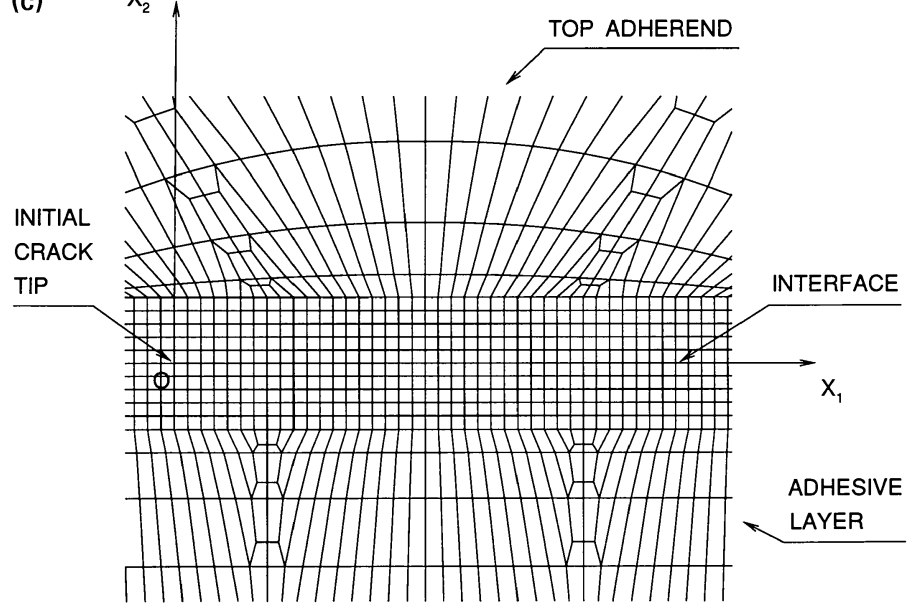

Figure 12. (a) Typical finite element mesh used for simulating interface crack growth in an adhesive joint under small-scale yielding conditions. (b) Details of the mesh near the interface region and the adhesive layer. (c) Details of the mesh near the crack tip. 
In order to control nodal instabilities that arise when a cohesive element loses its stress carrying capacity, the Ritz-finite element procedure, discussed in $\S 4$ is employed. As mentioned earlier, the novel feature of this procedure is that it enables controlling directly the relative separation of initially coincident nodes across the interface. The remote boundary conditions are prescribed according to the $K_{I}-K_{I I}$ field, but the increase in $|\mathbf{K}|=\left(K_{I}^{2}+K_{I I}^{2}\right)^{1 / 2}$ during incremental propagation of the crack is obtained from the analysis so as to maintain overall equilibrium of the finite element system. The rate of increase in $|\mathbf{K}|$ decreases with continued crack propagation and, finally, $|\mathbf{K}|$ approaches an asymptotic limit as steady-state crack growth conditions are attained.

In these simulations, the Drucker-Prager model (see Chen \& Han 1988) is employed to describe the constitutive response of the polymeric adhesive layer. The Drucker-Prager yield function is given as (Chen \& Han 1988):

$$
\Phi\left(\sigma_{i j}, \sigma^{c}\right)=\sqrt{3 J_{2}}+\sigma_{m} \tan \alpha-\left(1-\frac{1}{3} \tan \alpha\right) \sigma^{c}=0,
$$

where, $J_{2}$ is the second invariant of the deviatoric part of the Cauchy stress $\sigma_{i j}$, and $\sigma_{m}=\sigma_{k k} / 3$ is the hydrostatic stress. Further, $\sigma^{c}$ is the true yield stress in a uniaxial compression test and $\alpha$ is a pressure sensitivity index which is usually less than $23^{\circ}$ for polymers (Bowden \& Jukes 1972; Brown 1987; Quinson et al 1997). A complete description of the finite deformation elastic-plastic constitutive equations for the above model is given by Roy Chowdhury \& Narasimhan (2000b).

The true stress-strain response of the polymeric material in uniaxial compression is idealized here by a piecewise power hardening law of the form:

$$
\frac{\epsilon^{c}}{\epsilon_{o}^{c}}=\left\{\begin{array}{cl}
\sigma^{c} / \sigma_{o}^{c}, & \sigma^{c} \leq \sigma_{o}^{c} \\
\left(\sigma^{c} / \sigma_{o}^{c}\right)^{n}, & \sigma^{c}>\sigma_{o}^{c}
\end{array}\right.
$$

Here, $\sigma_{o}^{c}$ is the initial yield stress, $\epsilon_{o}^{c}=\sigma_{o}^{c} / E$ is the initial yield strain under uniaxial compression and $n$ is the strain hardening exponent of the material. The initial yield stress under uniaxial tension, $\sigma_{o}^{t}$, is related to $\sigma_{o}^{c}$ by,

$$
\sigma_{o}^{t} / \sigma_{o}^{c}=\left(1-\frac{1}{3} \tan \alpha\right) /\left(1+\frac{1}{3} \tan \alpha\right) .
$$

In the present analysis, $E / \sigma_{o}^{c}, n$, and Poisson's ratio $\nu$ are taken as 300, 10 and 0.36 respectively. The cohesive zone parameters $\lambda_{1}$ and $\lambda_{2}$ (see figure 1c) are chosen as 0.15 and 0.5 respectively. Further, the critical stress parameter $\hat{\sigma}$ is taken as $2 \sigma_{o}^{t}$ and $\delta_{n}^{c}=\delta_{t}^{c}$ is assumed.

\subsection{Results and discussion}

In this work, crack growth resistance is characterized by the effective stress intensity factor $|\mathbf{K}|$. In absence of plasticity in the adhesive layer, catastrophic failure takes place at $|\mathbf{K}|=K_{\circ}$, where,

$$
K_{o}=\left[E \Gamma_{o} /\left(1-\nu^{2}\right)\right]^{1 / 2} .
$$

On the other hand, energy dissipation through plastic deformation in the adhesive layer enhances the toughness of the system leading to an increase in $|\mathbf{K}| / K_{\circ}$ during the initial 
part of crack growth. Following Tvergaard \& Hutchinson (1996), a material length quantity, $R_{o}$, is defined as:

$$
R_{o}=\frac{2}{3 \pi\left(1-\beta^{2}\right)}\left[\frac{\left(1-\nu^{2}\right)}{E}+\frac{\left(1-\nu_{s}^{2}\right)}{E_{s}}\right]^{-1} \frac{\Gamma_{o}}{\left(\sigma_{o}^{t}\right)^{2}},
$$

where, $\beta$ is the second Dundurs elastic mismatch parameter given by,

$$
\beta=\frac{1}{2} \frac{\mu\left(1-2 \nu_{s}\right)-\mu_{s}(1-2 \nu)}{\mu\left(1-\nu_{s}\right)-\mu_{s}(1-\nu)} .
$$

Here, $\nu_{s}$ and $\nu$ are the Poisson ratios and $\mu_{s}$ and $\mu$ are the shear moduli of adherend and the layer respectively. The natural length scale $R_{o}$ is an approximate estimate of the size of the plastic zone at the initiation of crack propagation for $h \gg R_{\circ}$ (Tvergaard \& Hutchinson 1996).

Figure 13a shows the resistance curves for different thicknesses of the layer corresponding to mode I loading $\left(\psi=0^{\circ}\right)$ in a pressure-sensitive layer with $\alpha=20^{\circ}$. In this figure, the normalised resistance $|\mathbf{K}| / K_{\circ}$ is plotted as a function of normalised crack growth $\Delta a / R_{\circ}$. It can be observed from figure $13 \mathrm{a}$ that in all cases, $|\mathbf{K}| / K_{\circ}$ increases monotonically with $\Delta a / R_{\circ}$ until steady state conditions are reached. As already mentioned, this is attributed to plastic dissipation in the adhesive layer. The value of $\Delta a / R_{\circ}$ corresponding to the stage when steady state is attained is a measure of the maximum amount of normalised stable crack growth that can be sustained by the system under a load controlled fracture test.

It can be seen from figure $13 \mathrm{a}$ that the initial part of the $R$-curves coincide for all values of the normalised thickness $h / R_{\circ}$. This is because during this stage of crack growth the plastic zone emanating from the crack tip is small in size and does not feel the presence of the lower interface. However, at later stages of crack growth, the $R$-curves pertaining to different $h / R_{\circ}$ values deviate from each other. Further, during this phase the resistance to crack growth increases with increase in thickness, when $h$ is changed from $3.33 R_{\circ}$ to $10 R_{\circ}$. It is found from the analysis that the plastic zone at steady state corresponding to $\psi=0^{\circ}$
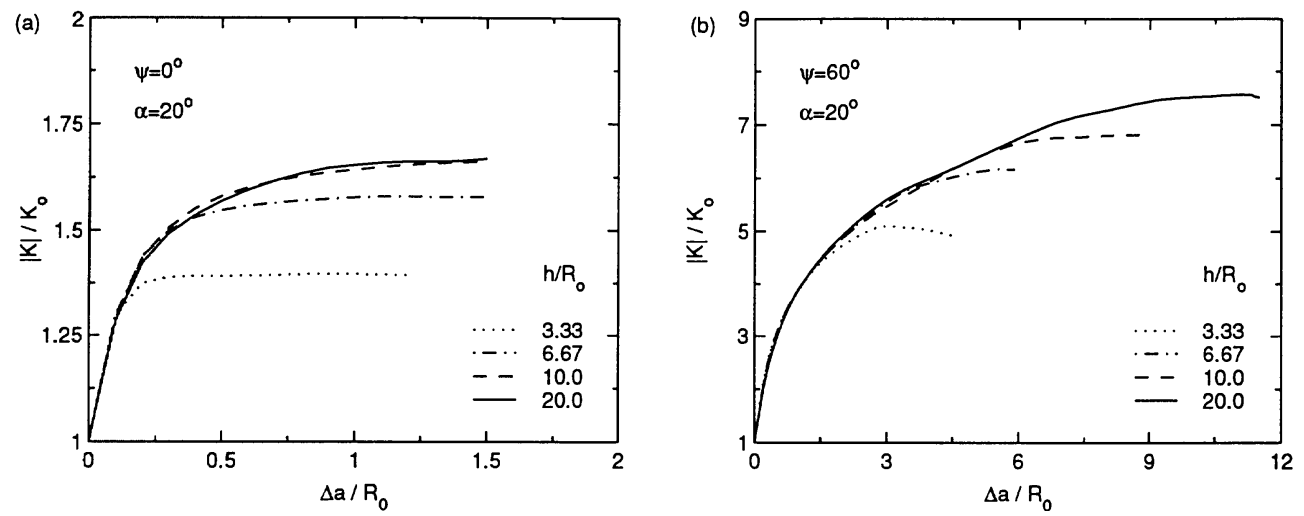

Figure 13. Normalized effective stress intensity factor $|\mathbf{K}| / K_{o}$ versus normalized crack extension $\Delta a / R_{o}$ ( $R$-curves) for different values of normalized layer thickness $h / R_{o}$ corresponding to a pressure sensitive layer with $\alpha=20^{\circ}$ under (a) mode I $\left(\psi=0^{\circ}\right)$ and (b) mixed-mode loading with $\psi=60^{\circ}$. 
encompasses the full thickness of the layer for low $h / R_{\circ}$ (see, Roy Chowdhury \& Narasimhan 2000a). Since the material properties are same, this implies that plastic dissipation would increase resulting in higher crack growth resistance as $h / R_{\circ}$ increases in the range $0<h / R_{\circ}<10$. On the other hand, in the range $h / R_{\circ} \geq 10$, the plastic zone at steady state does not extend up to the lower interface and is fully contained within the adhesive layer. In this range, the plastic zone size remains invariant with respect to layer thickness (Roy Chowdhury \& Narasimhan 2000a). Accordingly, the layer thickness plays no role and the $R$-curves pertaining to $h / R_{\circ}=10$ and 20 in figure 13a coincide with each other.

Figure $13 \mathrm{~b}$ shows the resistance curves corresponding to $\psi=60^{\circ}$ and $\alpha=20^{\circ}$. It can be observed from this figure that, unlike in the mode I case, resistance to crack growth increases with $h / R_{\circ}$ even up to $h / R_{\circ}=20$. This is because the steady state plastic zone for $\psi=60^{\circ}$ engulfs the full thickness of the layer even at $h / R_{\circ}=20$ (Roy Chowdhury \& Narasimhan 2000a). On comparing figures $13 \mathrm{a}$ and $\mathrm{b}$, it is found that the steady state fracture toughness is much higher for $\psi=60^{\circ}$ than for $\psi=0^{\circ}$, for the entire range of thickness considered. These trends match well with the experimental observations of Chai (1988) and Swadener \& Liechti (1998).

\section{Concluding remarks}

In this paper, the fundamental concepts and the finite element formulation of a cohesive zone model, employed to simulate the processes of fracture and delamination in solids in a direct manner, have been presented in detail. The Ritz-finite element procedure, a special solution technique which is necessary to control nodal instabilities that arise during the process of debonding along the cohesive zone has also been discussed. Single cohesive elements tests have been carried out in order to validate the implementation of the formulation. Further, some simple model problems have been solved in order to demonstrate the capabilities and the robustness of the Ritz procedure. It has been observed that, in contrast to the conventional finite element solution technique, the Ritz procedure is able to effectively capture the softening and unstable branch of the solution curve. Finally, the process of stable interfacial crack growth in an adhesively bonded system is simulated using the cohesive zone model. It is found that the trends in the computed crack growth resistance curves match well with experimental observations. In particular, the steady state fracture toughness is found to increase with thickness of the adhesive layer as well as with the mode mixity associated with the external loading.

The first author wishes to thank the Council of Scientific and Industrial Research, India for assistance provided through a Research Associateship (No. 9/79(695)/98-EMR-I). The second author would like to gratefully acknowledge the Indian Space Research Organisation for financial support through the sponsored project ISTC/ME/RN/90.

\section{References}

Amazigo J C, Hutchinson J W 1977 Crack-tip fields in steady crack growth with linear strainhardening. J. Mech. Phys. Solids 25: 81-97

Barenblatt G I 1962 The mathematical theory of equilibrium of crack in brittle fracture. Adv. Appl. Mech. 7: 55-129 
Bowden P B, Jukes J A 1972 The plastic flow of isotropic polymers. J. Mater. Sci. 7: 52-63

Brown N 1987 In Engineered materials handbook (ASM Int.) vol. 2, pp 730-731

Camacho G T, Ortiz M 1996 Computational modelling of impact damage in brittle materials. Int. J. Solids Struct. 33: 2899-2938

Chai H 1988 Shear fracture. Int. J. Fracture 37: 137-159

Chai H 1992 Micromechanics of shear deformations in cracked bonded joints. Int. J. Fracture 58: 223-239

Chai H, Chiang M Y M 1996 A crack propagation criterion based on local shear strain in adhesive bonds subjected to shear. J. Mech. Phys. Solids 44: 1669-1689

Chen W F, Han D J 1988 Plasticity for structural engineers (Berlin: Springer Verlag)

Chitaley A D, McClintock F A 1971 Elastic-plastic mechanics of steady crack growth under antiplane shear. J. Mech. Phys. Solids 19: 147-163

Drugan W J, Rice J R, Sham T L 1982 Asymptotic analysis of growing plane strain tensile cracks in elastic-ideally plastic solids. J. Mech. Phys. Solids 30: 447-473

Dugdale D S 1960 Yielding of steel sheets containing slits. J. Mech. Phys. Solids 8: 100-108

Gao G, Klein P 1998 Numerical simulation of crack growth in an isotropic solid with randomized internal cohesive bonds. J. Mech. Phys. Solids 46: 187-218

Hibbitt H D, Marcal P V, Rice J R 1970 A finite element formulation for problems of large strain and large displacement. Int. J. Solids Struct. 6: 1069-1086

Kanninen M F, Popelar C H 1985 Advanced fracture mechanics (Oxford: University Press)

Kolhe R, Tang S, Hui C Y, Zehnder A T 1999 Cohesive properties of nickel-alumina interfaces determined via simulations of ductile bridging experiments. Int. J. Solids Struct. 36: 5573-5595

Lin G, Kim Y J, Cornec A and Schwalbe K H 1997 Fracture toughness of a constrained metal layer. Comput. Mater. Sci. 9: 36-47

Malvern L E 1969 Introduction to the mechanics of a continuous medium. (Englewood Cliffs, NJ: Prentice Hall)

Malluck J F, King W W 1980 Fast fracture simulated by conventional finite elements: a comparison of two energy-release algorithms. In Crack arrest methodology and application (eds) G T Hahn, M F Kanninen (Philadelphia: Am. Soc. for Testing \& Mater.) ASTM STP 711, pp 38-53

McMeeking R M, Rice J R 1975 Finite element formulation for problems of large elastic-plastic deformation. Int. J. Solids Struct. 11: 601-616

Narasimhan R, Rosakis A J, Hall J F 1987a A finite element study of stable crack growth under plane stress conditions: Part I - Elastic-perfectly plastic solids. J. Appl. Mech. 54: 838-845

Narasimhan R, Rosakis A J, Hall J F 1987b A finite element study of stable crack growth under plane stress conditions: Part II - Influence of hardening. J. Appl. Mech. 54: 846-853

Needleman A 1982 Finite elements for finite strain plasticity problems. In Plasticity of metals at finite strain: Theory, experiment and computation (eds) E H Lee, R L Mallett

Needleman A 1987 A continuum model for void nucleation by inclusion debonding. ASME J. Appl. Mech. 54: 525-531

Quinson R, Perez J, Rink M, Pavan A 1997 Yield criteria for amorphous glassy polymers. J. Mater. Sci. 32: 1371-1379

Rice J R, Sorensen E P 1978 Continuing crack-tip deformation and fracture for plane strain crack growth in elastic-plastic solids. J. Mech. Phys. Solids 26: 163-186

Roychowdhury S, Narasimhan R 2000a A finite element analysis of quasistatic crack growth in a pressure sensitive constrained ductile layer. Engng. Fract. Mech. 66: 551-571

Roychowdhury S, Narasimhan R 2000b A finite element analysis of stationary crack tip fields in a pressure sensitive constrained ductile layer. Int. J. Solids Struct. 37: 3079-3100

Sham T L 1983 A finite element study of the asymptotic near-tip fields for mode I plane strain cracks growing stably in elastic-ideally plastic solids. Elastic-plastic fracture: Second symposium, Volume I - Inelastic crack analysis, (eds) C F Shih, J P Gudas (Philadelphia: Am. Soc. for Testing \& Mater.) ASTM STP 803, pp 52-79 
Swadener J G, Liechti K M 1998 Asymmetrical shielding mechanism in the mixed-mode fracture of a glass/epoxy interface. ASME J. Appl. Mech. 65: 25-29

Tadmor E B, Ortiz M, Phillips R 1996 Quasicontinuum analysis of defects in solids. Philos. Mag. A73: $1529-1563$

Taylor R L 1979 Computer procedures for finite element analysis. The finite element method (ed.) O C Zienkiewicz, 3rd edn (New Delhi: Tata McGraw Hill) chap. 24

Tvergaard V, Hutchinson J W 1992 The relation between crack growth resistance and fracture process parameters in elastic-plastic solids. J. Mech. Phys. Solids 40: 1377-1397

Tvergaard V, Hutchinson J W 1993 The influence of plasticity on mixed mode interface toughness. $J$. Mech. Phys. Solids 41: 1119-1135

Tvergaard V, Hutchinson J W 1994 Toughness of an interface along a thin ductile layer joining elastic solids. Philos. Mag. A70: 641-656

Tvergaard V, Hutchinson J W 1996 On the toughness of ductile adhesive joints. J. Mech. Phys. Solids 44: 789-800

Xia L, Shih C F, Hutchinson J W 1995 A computational approach to ductile crack growth under large scale yielding conditions. J. Mech. Phys. Solids 43: 389-413 